\title{
Postoperative Acute Lumbar Epidural Hematoma With White Clot Formation Causing Cauda Equina Syndrome: A Case Report and Review of the Literature
}

\author{
Ioannis D. Gelalis ${ }^{\mathrm{a}, \mathrm{c}}$, Angelos N. Politis ${ }^{\mathrm{a}}$, Christina M. Arnaoutoglou ${ }^{\mathrm{a}}$, Athanasios C. Karageorgos ${ }^{\mathrm{a}}$, \\ Nikolaos K. Paschos ${ }^{\mathrm{a}}$, Avraam Ploumis ${ }^{\mathrm{b}}$, Theodoros A. Xenakis ${ }^{\mathrm{a}}$
}

\begin{abstract}
While the majority of spinal epidural hematomas after a spinal operation are clinically asymptomatic, the reported percentage of hematomas needing surgical evacuation is only $0.1 \%$. A 69 -year old woman presented after a rapidly progressive worsening of her neurologic status at both legs. The patient was under anticoagulant medication with acenocoumarol due to atrial fibrillation and underwent a decompression laminectomy and L5, S1 left nerve root decompression. Eleven hours after the operation she presented with acute progressive cauda equina syndrome due to an epidural hematoma and was immediately transferred to the operating theater. A membrane of white clot was found and the presence of epidural clotted blood beneath the membrane was verified. The surgeon must be aware of this catastrophic complication that requires a high level of suspicion and prompt intervention. Large, multicentre prospective, randomized studies are needed to fully investigate the risks of anticoagulation therapy after spinal surgery.
\end{abstract}

Keywords: White clot; Epidural hematoma; Anticoagulation; Lumbar spinal stenosis; Laminectomy

\footnotetext{
Manuscript accepted for publication December 7, 2011

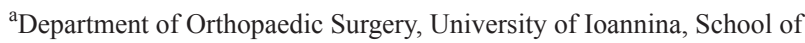
Medicine, Ioannina, Greece

${ }^{b}$ Department of Rehabilitation, University of Ioannina, School of Medicine, Ioannina, Greece

${ }^{\mathrm{c} C}$ Correspondence author: Ioannis D. Gelalis, Department of

Orthopaedic Surgery, University of Ioannina, School of Medicine,

Pantazidi 11 Str., Ioannina, 45445, Greece.

Email: idgelalis@gmail.com

doi: $10.4021 / \mathrm{jmc} 462 \mathrm{w}$
}

\section{Introduction}

Although a spinal epidural hematoma usually occurs after a spinal operation, the majority are clinically asymptomatic [1]. Rarely, they can compress the spinal cord or nerve roots significantly and require immediate surgical intervention. The neurological consequences can be devastating and include peri-incisional pain, paresthesia, radicular pain, bladder dysfunction and bilateral neurologic deficits.

The specific use of anticoagulants such as low molecular weight heparin for the prophylaxis of deep vein thrombosis, treatment of pulmonary embolism or cardiac conditions such as atrial fibrillation is currently increasing. Anticoagulation after a major spinal surgery is of concern, because of the possibility of excessive bleeding or postoperative hematomas and associated neurologic deficits [2]. Moreover, spontaneous epidural hematoma has been reported in association with anti-thrombotic therapy with heparin [3].

We present a case of a 69-year old woman with a history of atrial fibrillation that was already under anticoagulation at the day of surgery. The patient developed acute cauda equina syndrome eleven hours postoperatively, due to a lumbar epidural hematoma, presenting as a white clot intraoperatively.

\section{Case Report}

A 69-year old woman with a history of chronic claudication and low back pain, presented in the emergency department after a rapidly progressive worsening of her neurologic status, characterized by left drop foot (MRC grade 1) and sensory loss of the dermatomes L4, L5 and S1 of her right leg associated with motor deficit (MRC grade 3). She presented with Oswestry Disability Index score $44 \%$ and Visual Analog Scale score for back and leg pain 6 out of 10. The patient was obese with BMI $=52.7$ and also had a history of hypertension, atrial fibrillation, rheumatoid arthritis and amiodarone pneumonitis. The patient was under anticoagulant medication with acenocumarol at the day of admission.

Plain x-rays revealed degenerative lumbar disc disease and loss of lumbar lordosis. Magnetic Resonance Imaging 


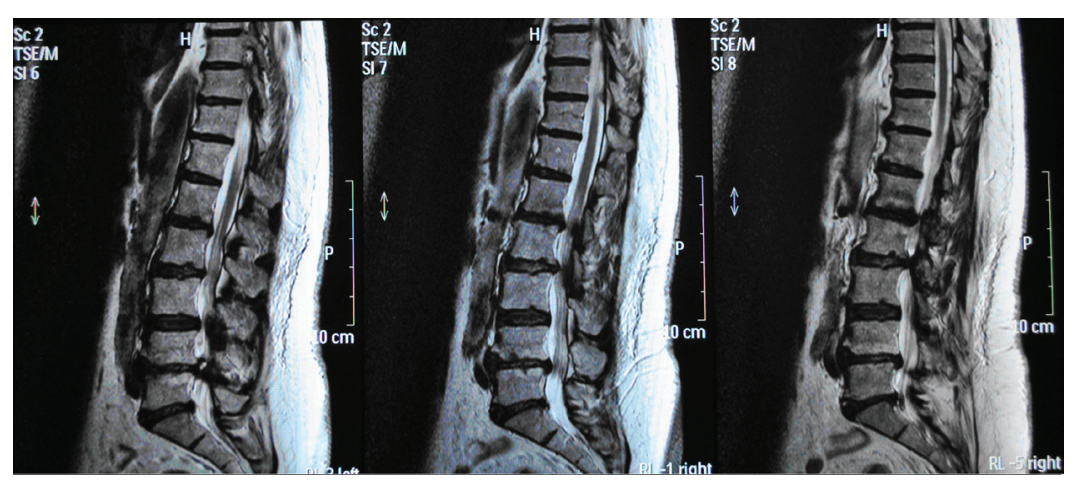

Figure 1. Pre-operative MRI scan of the patient.

(MRI) of the lumbar spine showed central and lateral spinal stenosis from L2 to S1, severe degenerative facet arthritis and multiple disc degeneration at the levels L2/L3, L3/L4, L4/L5 and L5/S1 (Fig.1). Electrophysiologic examination revealed active neurological compression mainly of the left L5 nerve root.

Due to the history of atrial fibrillation treated with acenocumarol and under strict instruction of our cardiology department the administration of acenocumarol was interrupted and the patient started treatment with LMWH (Enoxaparin $120 \mathrm{mg}$ /day). Prior to surgery the following blood values were measured: PT $23.6 \mathrm{sec}$, APTT 33.1 and INR 1.79, HCT $42.9 \%, \mathrm{Hb} 9.9 \mathrm{gr} / \mathrm{dl}$ and PLT $236000 / \mu \mathrm{l}$.

Our preoperative plan included decompression of the L2-S1 spine levels and restoration of the sagittal balance of the lumbar spine via posterior instrumentation with pedicle screws and rods. In the operating theater just after general anesthesia was administrated, the anaestesiologist noted that the patient had difficulty to maintain high levels of SatO2, due to the amiodarone related lung disease and the time of the operation should be as short as possible. Despite our preoperative plan, the patient underwent a decompression laminectomy at levels L3, L4, L5 and S1 and L5, S1 left nerve root decompression. Blood loss during the operation was $700 \mathrm{cc}$ and the total duration of the operation was 1 hour and 45 minutes.

Eleven hours after the operation the patient presented with acute progressive cauda equina syndrome with sensory deficit and severe motor deficit in the distribution of L5 and S1 nerve roots of her both legs and bladder dysfunction. An emergency MRI scan revealed the presence of an epidural hematoma severely compressing dura at L4, L5 and S1 levels (Fig. 2, 3). The patient was immediately transferred to the operating theater 4 hours after the presentation of the acute cauda equina syndrome. At the revision operation, a membrane of white clot was found in the operation site and the presence of epidural clotted blood beneath the membrane was verified. The white clot and the underlying epidural blood clot were carefully removed. No active bleeding was noted.

After the second operation blood values of HCT $31.1 \%$,

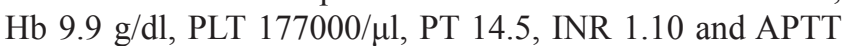
22.5 were measured. At the first postoperative day, enoxaparin $120 \mathrm{mg}$ /day was replaced with tinzaparin 4500 antiXa

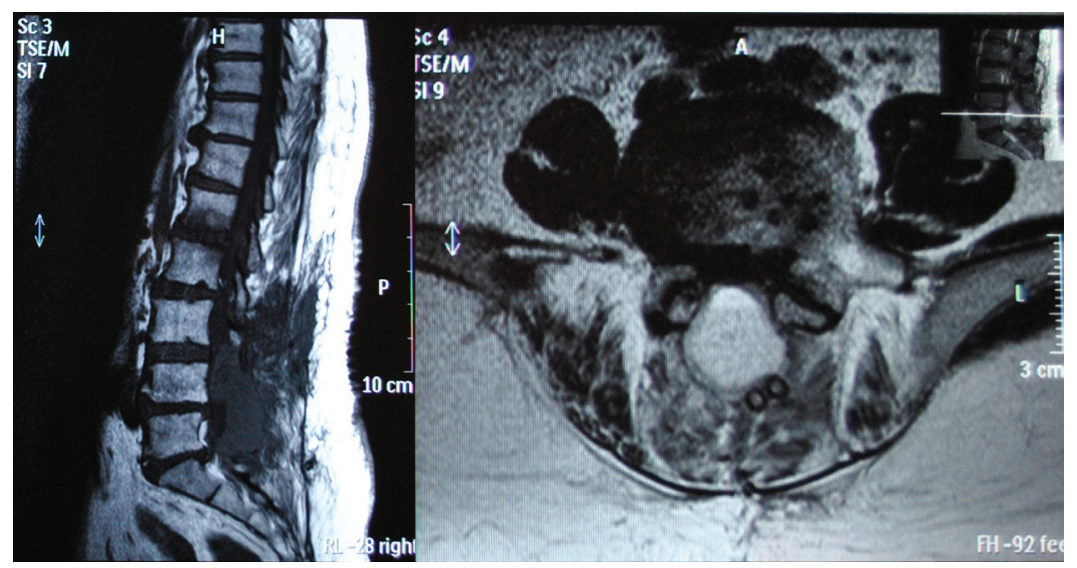

Figure 2. Post-operative MRI scan showing the presence of the epidural hematoma. Note the suction tubes over the hematoma mass. 


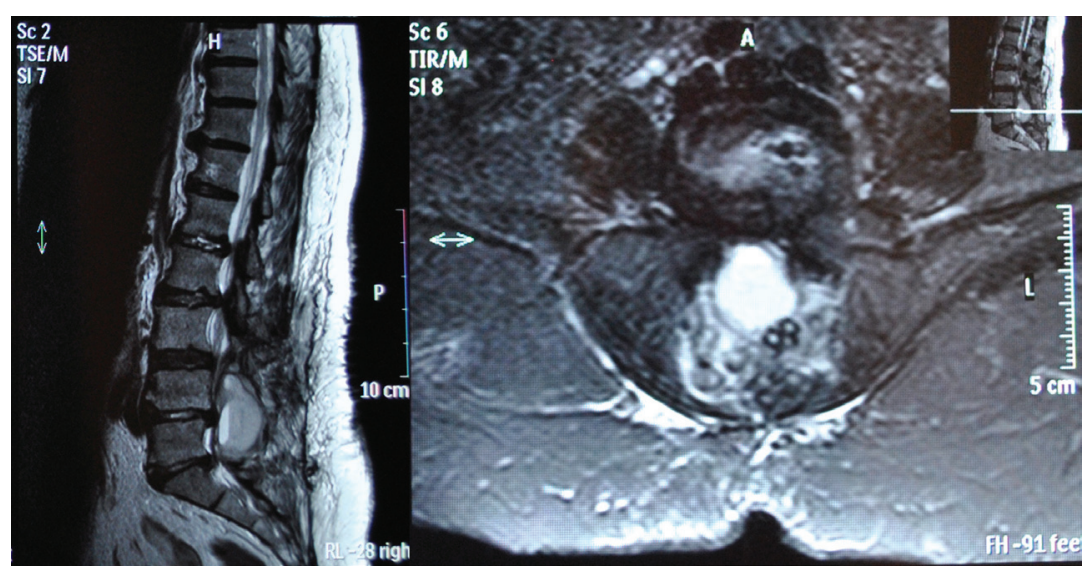

Figure 3. Post-operative MRI scan showing compression of dura at L4, L5 and S1 lev els from the sizeable epidural hematoma.

IU/0.45 $\mathrm{ml}$. The postoperative course of the patient was uneventful. Eight months after the revision operation the patient has almost recovered, but still presents with decreased sensation in the L5 and S1 dermatomes of the left leg with left drop foot as prior to the operation and occasional mild low back pain.

\section{Discussion}

Although rare, the experience of a new onset major neurologic deficit after spinal surgery is devastating for the patient. The incidence of significant spinal cord or cauda equina injury after spinal surgery for specific disease entities ranges from approximately $0 \%$ to $2 \%$. The overall incidence of new onset major neurologic deficit immediately after spinal surgery in the lumbar/sacral spine was $0.0745 \%$ in a retrospective study by Cramer et al [4].

One of the causes of neurologic injury during and after spinal surgery is compressive spinal epidural hematomas. Causes of spinal epidural hematomas include trauma (including vertebral fractures), spinal surgery, spinal epidural procedures, anticoagulation therapy, vertebral hemangiomas, vascular anomalies such as arteriovenous malformation and blood dyscrasias.

Kebaish et al. investigated in a retrospective study the significance of individual factors related to the formation of spinal epidural hematomas. Preoperative variables that are significant risks for postoperative hematomas include an age older than 60 years, the use of preoperative non-steroidal anti-inflammatory drugs and a Rh-positive blood type. Intraoperative variables that proved to be significant include more than five spinal levels subjected to surgery, hemoglobin level less than $10 \mathrm{~g} / \mathrm{dl}$, and blood loss of more than $1 \mathrm{~L}$. Length of surgery, requiring fresh-frozen plasma or platelets, and the use of a cell saver are not significant risk factors. Postoperatively, significant variables include prothrombin time and international normalized ratio greater than 2 within the first 48 hours. The use of well-controlled anticoagulation therapy for DVT and/or cardiovascular prophylaxis, and subfascial drains are not directly associated with the development of postoperative spinal epidural hematomas [5]. Kou et al. found that multilevel procedures and preoperative coagulopathy are statistically significant predictors of symptomatic epidural hematomas, while age, body mass index, peri-operative durotomies and post-operative drains were not statistically significant risk factors [6].

Asymptomatic epidural hematomas have been identified in $33 \%$ to $100 \%$ of patients after lumbar spine surgery, as shown on Computed Tomography or Magnetic Resonance Imaging studies [7]. The reported percentage of hematomas needing surgical evacuation is only $0.1 \%$ [8]. Several series estimate the incidence of cauda equina syndrome after lumbar spine surgery between 0.1 and $0.2 \%[5,9]$.

Despite its rarity, a postoperative spinal epidural hematoma has the potential to result in devastating neurologic sequelae and requires the continued vigilance of the spine surgeon. Most spinal epidural hematomas occurring following spinal surgery are diagnosed within 24 hours. Symptoms can be acute or delayed. Delayed are defined as neurologic deterioration more than 3 days after operation [10]. Post-operative symptoms progress from sharp, severe peri-incisional pain to paresthesia, radicular pain, bladder dysfunction and bilateral neurologic deficits, such as motor weakness and sensory loss.

Rapid diagnosis is crucial for prompt surgical intervention. Magnetic Resonance Imaging is the investigation method of choice and has replaced myelography as the screening test for diagnosis. In cases where the method cannot be safely performed, as in patients with instrumentation after spinal fusion, Computed Tomography with 3D reconstruction protocols can help. The postoperative appearance of the hematoma has been described as an area of isointense or increased signal intensity on T1-weighted images and heterog- 
enous hyperintense on T2-weighted images in the epidural space. The sagittal and parasagittal images usually show a convex lens-shaped lesion [11].

Urgent decompression of all involved spinal levels, preferably within 6 hours, aids in neurological recovery and is the treatment of choice. Laminectomy is performed to decompress the entire length of the compressed spinal canal and remove the coagulated products. If active epidural bleeding is found, it should be cauterized using bipolar electrocautery [5]. Prognosis can depend on several factors such as the rate of development of symptoms, time to surgical decompression, spinal level involvement, and the degree of neurologic deficit $[6,8]$.

Batson's internal plexus of veins is reported as the source of bleeding in the formation of the hematomas. It is also postulated that rupture of valveless veins in the internal vertebral plexus, even by the slightest change of posture during sleep, turning, coughing, straining or due to Valsalva's maneuver, can cause epidural bleeding [12]. The source of bleeding can also be arterial, from paraspinal muscular branches.

To our knowledge, this is the only reported case of a visible white clot formation in the revision decompression surgery due to a spinal epidural hematoma. The formation of a white clot is the first stage of blood clotting. Activated platelets begin adhering to the wall of the blood vessels at the site of bleeding and within a few minutes the white clot is formatted. The authors attribute the presence of the white clot membrane to the first phase of blood clotting, while the mature red blood clot was formatted underneath the prementioned membrane.

Low-molecular-weight heparin (LMWH) is increasingly used to provide anticoagulation in preventative and therapeutic settings. As far as morbidity of LMWH in spinal surgery is concerned, Gerlach et al. found a $0 \%$ incidence of pulmonary embolism and a $0.05 \%$ incidence of deep venous thrombosis using this type of prophylaxis in a large cohort of patients [13]. Nevertheless, the catastrophic morbidity of a symptomatic postoperative epidural hematoma remains a substantial disincentive to start chemoprophylaxis after spinal surgery and little is known about the exact risks of full anticoagulation therapy in spinal surgery. The aforementioned study reported a very low incidence of post-operative hematomas $(0.7 \%)$.

In a systematic review Glotzbecker et al. concluded that there is insufficient published data available to precisely define the safety of postoperative chemoprophylaxis. The available evidence does suggest that use of therapeutic doses of heparin in postoperative spinal patients who sustain a pulmonary embolism may have a higher incidence of bleeding complications [9]. Barnes et al. in a literature review concluded that there is insufficient data to establish evidencebased guidelines for the use of heparin or an equivalent anticoagulation protocol in patients who have recently un- dergone spinal surgery. The authors suggest that in patients who undergo spinal surgery and who require heparinization therapy for myocardial ischemia or infarction, the use of frequent neurological examinations in conjunction with anticoagulation therapy seems to be the only reasonable option [14].

Cheng et al. in a recent systematic review concluded that chemical prophylaxis should be considered in spine trauma patients that are at increased risk. The authors also agree that the safe timing of administration of anticoagulation agents is unknown [2]. According to the Sixth ACCP Consensus Conference on Antithrombotic Therapy, no firm recommendations exist for elective spine surgery. It is reasonable to use elastic stockings alone, low-dose unfractionated heparin alone, or the combination of the two; intra-operative plus postoperative intermittent pneumatic compression, may also be effective. For spine surgery patients with additional thromboembolic risk factors, prophylaxis with one of these options is suggested [15].

This case is consistent with an extended postoperative epidural hematoma after decompression-laminectomy in a patient under full anticoagulation therapy for atrial fibrillation. It also points the risks of anticoagulation therapy in spine surgery. The essential steps in diagnosis and treatment are analyzed. The surgeon must be aware of this catastrophic complication that requires a high level of suspicion and prompt intervention. Research is currently underway in the field of anticoagulation therapy that may change the way such medications are used. Low molecular weight heparin is currently the gold standard in treatment protocols for thromboembolic disease and cardiac conditions. The use of full anticoagulation should be used cautiously in the early postoperative period. Large, multicentre prospective, randomized studies are needed to fully investigate the associated risks and consequences of anticoagulation therapy after spinal surgery. Lacking specific guidelines, each patient should be treated on an individual basis, and the potential benefits of anticoagulation should be compared with the potential risks (risk-benefit ratio). The growing field of spine surgery today includes many surgical procedures for a variety of pathologies, and involves a highly heterogeneous class of patients. There is no unique risk factor, because spinal surgery does not take one single form and that it is therefore not possible to suggest a standardized thromboprophylaxis for spinal surgery, as can be doness for hip and knee surgery. Any neurologic change requires rapid diagnostic imaging and prompts surgical decompression to arrest and reverse the progression of the injury.

\section{Conflict of Interest Statement}

The authors report no actual or potential conflict of interest in relation to this article. 


\section{Funding}

None

\section{References}

1. Teplick JG, Haskin ME. Review. Computed tomography of the postoperative lumbar spine. AJR Am J Roentgenol. 1983;141(5):865-884.

2. Cheng JS, Arnold PM, Anderson PA, Fischer D, Dettori JR. Anticoagulation risk in spine surgery. Spine (Phila Pa 1976). 2010;35(9 Suppl):S117-124.

3. Gaiser RR, Berkowitz DH, Chou D. Epidural blood patch in a patient taking enoxaparin. J Clin Anesth. 2004;16(5):386-388.

4. Cramer DE, Maher PC, Pettigrew DB, Kuntz Ct. Major neurologic deficit immediately after adult spinal surgery: incidence and etiology over 10 years at a single training institution. J Spinal Disord Tech. 2009;22(8):565-570.

5. Kebaish KM, Awad JN. Spinal epidural hematoma causing acute cauda equina syndrome. Neurosurg Focus. 2004;16(6):e1.

6. Kou J, Fischgrund J, Biddinger A, Herkowitz H. Risk factors for spinal epidural hematoma after spinal surgery. Spine (Phila Pa 1976). 2002;27(15):1670-1673.

7. Ikuta K, Tono O, Tanaka T, Arima J, Nakano S, Sasaki K, Oga M. Evaluation of postoperative spinal epidural hematoma after microendoscopic posterior decompression for lumbar spinal stenosis: a clinical and magnetic resonance imaging study. J Neurosurg Spine. 2006;5(5):404-
409.

8. Lawton MT, Porter RW, Heiserman JE, Jacobowitz R, Sonntag VK, Dickman CA. Surgical management of spinal epidural hematoma: relationship between surgical timing and neurological outcome. J Neurosurg. 1995;83(1):1-7.

9. Glotzbecker MP, Bono CM, Wood KB, Harris MB. Postoperative spinal epidural hematoma: a systematic review. Spine (Phila Pa 1976). 2010;35(10):E413-420.

10. Uribe J, Moza K, Jimenez O, Green B, Levi AD. Delayed postoperative spinal epidural hematomas. Spine J. 2003;3(2):125-129.

11. Djukic S, Vahlensieck M, Resendes M, Genant HK. The lumbar spine: postoperative magnetic resonance imaging. Bildgebung. 1992;59(3):136-146.

12. Groen RJ, Ponssen H. The spontaneous spinal epidural hematoma. A study of the etiology. J Neurol Sci. 1990;98(2-3):121-138.

13. Gerlach R, Raabe A, Beck J, Woszczyk A, Seifert V. Postoperative nadroparin administration for prophylaxis of thromboembolic events is not associated with an increased risk of hemorrhage after spinal surgery. Eur Spine J. 2004;13(1):9-13.

14. Barnes B, Alexander JT, Branch CL, Jr. Postoperative Level 1 anticoagulation therapy and spinal surgery: practical guidelines for management. Neurosurg Focus. 2004;17(4):E5.

15. Geerts WH, Heit JA, Clagett GP, Pineo GF, Colwell CW, Anderson FA, Jr., Wheeler HB. Prevention of venous thromboembolism. Chest. 2001;119(1 Suppl):132S$175 \mathrm{~S}$ 